tions between the parameters defining the system. Finally, the conditions determined by three relations form the third group and are all conditions of constraint without friction.

This general theory is followed by numerous applications worked out completely, and with the elegance and clearness which are characteristic of the two courses of lectures we have before us.

It is only fair in concluding this review to remark to $\mathrm{Mr}$. Hermann's credit that the reading of these two volumes is not in the slightest degree trying to the eyes, which unfortunately could not be said with regard to, for instance, $\mathrm{Mr}$. Klein's lithographed courses.

JoHNs HopkINS UNIVERSITY,

Alexandre S. Chessin. January 24, 1896.

\title{
A GEOMETRIC PROOF OF A FUNDAMENTAL THEOREM CONCERNING UNICURSAL CURVES.
}

BY PROFESSOR W. F. OSGOOD.

1. IF $f(x, y)=0$ is the equation of an irreducible curve of deficiency 0 , then, as is well known, the coördinates can be expressed as rational functions of a parameter $\lambda: *$

$$
x=r_{1}(\lambda) \quad y=r_{2}(\lambda)
$$

where not only to a given value of $\lambda$ corresponds one and only one point of the curve, but conversely to a given point $(x, y)$ on the curve corresponds in general one and only one value of $\lambda . \dagger \quad \lambda$ can be expressed as a rational function of $x$ and $y$.

If a multiple-leaved Riemann surface spread out, say, over the $x$-plane be used to represent geometrically the above locus, $f(x, y)=0$, the deficiency of this surface will likewise be 0 , and as is shown in the elements of Riemann's theory of functions, $\$$ there exist single-valued functions on such a surface having but a single pole, and that of the first order, and taking on every value once and only once on the surface. Call such a function $\lambda$. Being single-valued on the surface it will be a rational function of $x$ and $y: \lambda=R(x, y)$,

\footnotetext{
*Salmon, Higher Plane Curves, p. 30; Clebsch-Lindemann, Geometrie vol. I., p. 883 .

† CAYLEY has given to such curves the name unicursal.

t KLein, Modulfunctionen, vol. I., p. 493 et seq. PICARD, Traité d' analyse, vol. II., Ch. XVI.
} 
and hence through this equation the multiple-leaved surface is transformed conformally into the single-leaved $\lambda$-plane. Hence $x$ is a rational function of $\lambda$. And similarly for $y$.

2. Conversely, let the equations

$$
\left.\begin{array}{l}
x=r_{1}(\lambda) \\
y=r_{2}(\lambda)
\end{array}\right\}
$$

be given, where $r_{1}$ and $r_{2}$ are any rational functions of $\lambda$ Then the locus of $(x, y)$, if regarded as a plane curve, is an algebraic irreducible curve of deficiency 0 ; if represented by a multiple-leaved surface spread out over the $x$-plane, it gives rise to an algebraic surface consisting of a single piece, likewise of deficiency 0 . (In connection with these statements see however the last lines of $\$ 5$.) But while to every value of $\lambda$ there still corresponds one and only one point $(x, y)$ of this surface, it is not true conversely that to a point $(x, y)$ only one value of $\lambda$ corresponds, as is shown by the example $x=\lambda^{2}, y=\lambda^{2}$. It is however always possible to replace $\lambda$ by a new parameter $\mu$, which is a certain rational function of $\lambda$ :

$$
\mu=r(\lambda)
$$

such that $x$ and $y$ will be rational functions of $\mu$, while to a given point $(x, y)$ only one value of $\mu$ corresponds.*

3. The essential thought that underlies these theorems finds clear expression in the geometric method of conformal representation, and reciprocally the value of this method as a means of investigation is set in a strong light.

It is believed that it will not be without interest to the readers of the Bulletin to see this simple example of the method worked out, and it is hoped furthermore that this example may be suggestive of a desirable for $m$ of presentation in instruction in the theory of functions.

4. Consider first the equation

$$
x=r_{1}(\lambda)
$$

To each value of $\lambda$ corresponds one and only one value of $x$ and the $\lambda$-surface consists of the single-leaved $\lambda$-plane. To each value of $x$ correspond $m$ values of $\lambda$, in general distinct, and the $x$-surface consists of an $m$-leaved Riemann surface connected through junctions along junction-lines. Call this surface $T$.

Now consider the second equation(1):

$$
y=r_{2}(\lambda) \text {. }
$$

$y$ will be a single-valued function on the surface $T$, since to

\footnotetext{
* An algebraic proof of this latter theorem was given by Lüroth, Math Annalen, vol. 9, 1875.
} 
every point of $T$ corresponds only one value of $\lambda$. But it may happen that for every value of $x$ two or more of the $m$ corresponding values of $y$ coincide; so that the surface $T$ would not represent $y$ as a function of $x$.

Let us construct the surface that does represent $y$ as a function of $x$. For this purpose first cut the leaves of $T$ along the junction lines, thus obtaining $m$ separate leaves, each containing one or more slits. Consider the first of these leaves. To each of its points corresponds a value of $y$, $y_{1}$, and it forms the first leaf of the surface to be constructed, $S$. Next let $x$ leave this first leaf and crossing one of the slits, pass into a second leaf of $T$. Call the corresponding value of $y$ in this second leaf $y_{2}$. Two cases can arise. Either $y_{2}$ is, in general, different from $y_{1}$ or $y_{2}$ is identical with $y_{1}$. In the first case this second leaf of $T$ is to be connected with the first leaf along this slit and thus forms the second leaf of $S$. In the second case the two edges of the slit in the first leaf are to be joined with each other and this slit thus disappears from $S$; $*$ the second leaf of $T$ does not belong to $S$. The example already cited will serve here as illustration: $x=\lambda^{2}, \quad y=\lambda^{2}$. The surface $T$ consists of two leaves with junctions at 0 and $\infty$; the junction-line may be taken as the positive axis of reals. The points of the first leaf shall represent those pairs of values $(x, \lambda)$ that flow continuously out of the pair $x=-1, \lambda=i$ when $x$ describes this leaf; $y_{1}$ is thus equal to $x$. When $x$ crosses the slit and thus passes into the second leaf of $T, y_{1}$ goes over into $y_{2}$. But $y_{2}=y_{1}$, both being equal to $x$, and hence in the construction of $S$ this slit disappears from the first leaf. In this example this is the only slit in the first leaf and thus the surface that represents $y$ as a function of $x$ is the singleleaved $x$-plane.

Returning now to the general case, we treat the other slits in the first leaf, if there be any, in the same way and thus finally either all the slits are closed and the first leaf forms the whole surface $S$, or else this first leaf is connected with other leaves along at least one slit. Such leaves will surely belong to the surface $S$. These leaves may be already connected along their slits with the first leaf, as in the case

$$
x=\lambda^{6}, \quad y=\lambda^{3} ;
$$

or perhaps it remained merely to join certain pairs of them

\footnotetext{
* It may happen that a slit, beside abutting on the two junctions at its extremities, passes through one or more junctions in its course. In that case I shall consider each of the segments of the slit as a separate slit.
} 
along certain slits, as in the case

$$
x=\lambda^{6}, \quad y=\lambda^{2}
$$

or it may be that one of these leaves contains a slit such that when the point $(x, y)$ crosses this slit, $y$ takes on still new values. Then these two leaves are to be joined along this slit and another leaf is added, as in the case

$$
x=\lambda^{6}, \quad y=\lambda \text {. }
$$

Proceeding in this way we finally obtain a closed surface $S$. This surface may or may not be the same as $T$. If it is, then it is of deficiency 0 , being conformally related to the single-leaved $\lambda$-surface. $\lambda$ is a single-valued function on this surface having no essentially singular points, and hence it is a rational function of $x$ and $y: *$

$$
\lambda=R(x, y) \text {. }
$$

Thus all the statements made in $\$ 2$ are proven.

5 . If, however, only a part of the leaves severed from $T$ have been incorporated into $S$, then it must be that in the construction of $S$ as described above, a leaf $L^{\prime}$ was found in which the values of $y$ were identical, for the same values of $x$, with values in another leaf $L$ already belonging to $S$; and hence $L^{\prime}$ was not joined to $S$, the connection along the corresponding slit being between the leaves already in $S$.

Consider now the leaf $L^{\prime}$. The values of $y$ in this leaf are the same for the same values of $x$ as in $L$. If we proceed to construct the Riemann surface for $y$ as a function of $x$, starting with the leaf $L^{\prime}$, we shall obviously be led to a surface $S^{\prime}$ identical with $S$; thus $S$ is repeated. If the leaves severed from $T$ are not yet exhausted, it will be possible to construct from them still a third surface $S^{\prime \prime}$, likewise identical with $S$. And so on. Thus finally the leaves of $T$ will have been combined in a set of $\rho$ surfaces, $S, S^{\prime \prime}, S^{\prime \prime} \ldots S^{(\rho-1)}$, each identical with the first, $S$. $\rho$ will divide $m$. Let $m=$ $m^{\prime} \rho$. Then $m^{\prime}$ is the number of leaves in $S$. The examples already cited illustrate clearly what has just been said.

So much for the surface $T$ and the surfaces $S$. Let us turn now to the corresponding regions of the $\lambda$-plane. To each of the $m$ leaves of the surface $T$ corresponds a region of the $\lambda$-plane, regions corresponding to leaves that are connected bordering on each other. If we recall the way in which the $S$-surface was generated by the successive addition of new leaves, we see that the counterpart of this process in the $\lambda$-plane consists in starting with the region

* Harkness and MorLey, Theory of Functions, \& 174; KLEIN-FrICKE, Modulfunctionen, vol. I., p. 499. 
corresponding to the first leaf of $S$ and then adding on bordering regions corresponding to the subsequent leaves of $S$, the final result being a connected region $N$, made up of $m^{\prime}$ of the former regions, which corresponds point for point and conformally to $S$. To $S^{\prime}$ will correspond a second such region $N^{\prime}$, to $S^{\prime \prime}$ a third, $N^{\prime \prime}, \ldots$ to $S^{(\rho-1)}, N^{(\rho-1)}$, and these $\rho$ regions will just cover the $\lambda$-plane.

Let $\lambda_{1}$ be any point lying in the region $N$. To $\lambda_{1}$ corresponds the point $(x, y)$ of each of the surfaces $S, S^{\prime}, \cdots S^{(\rho-1)}$ and to these points correspond in turn values $\lambda_{1}{ }^{\prime}, \lambda_{1}{ }^{\prime \prime}, \cdots \lambda_{1}{ }^{(\rho-1)}$ belonging respectively to $N^{\prime}, N^{\prime \prime}, \cdots N^{(\rho-1)}$. Let us from now on, discarding the surfaces $S^{\prime \prime}, S^{\prime \prime}, \cdots S^{(\rho-1)}$, restrict ourselves to $S$, so that $\lambda^{\prime}{ }_{1}, \lambda_{1}{ }^{\prime \prime}, \cdots \lambda_{1}{ }^{(p-1)}$ will now be regarded as functions of $(x, y)$ on $S$. Thus any region of $S$ that does not contain any part of one of the slits that has been closed up or along whose edges the sheets have been connected differently in $S$ from in $T$ will be transformed conformally on a region of each of the domains $N, N^{\prime}, \cdots N^{(\rho-1)}$. If the point $(x, y)$, moving on $S$, cross one of the slits just excepted, then, since this slit corresponds to the boundary between $N^{(i)}$ and $N^{(k)}, \lambda_{1}^{(i)}$ will cross this boundary and enter the region $N^{(k)}$, another one of the $\lambda_{1}$ 's entering at the same time the region $N^{(i)}$ just vacated by $\lambda_{1}{ }^{(i)}$. And if $(x, y)$ describe any closed path on $S$, the $\lambda_{1}$ 's will either describe closed paths or be permuted. Now form the symmetric product

$$
\mu=\lambda_{1} \lambda_{1}{ }^{\prime} \lambda_{1}{ }^{\prime \prime} \ldots \lambda_{1}^{(\rho-1)} \text {. }
$$

This function is in general monogenic on $S$, since this is true of each of the factors (on account of the conformal correspondence), and furthermore it is single-valued. It has no essentially singular point and is therefore a rational function of $x$ and $y$,

$$
\mu=R(x, y) \text {. }
$$

The point $\lambda=\infty$ will lie in general entirely within one of the regions $N$, so that only one factor will ever become infinite, and this factor will afford a pole of the first order. No other factor becoming 0 simultaneously, $* \mu$ has but one pole and that of the first order, so that it takes on every value once and only once on $S$.

It thus appears that the surface $S$ is transformed conformally on a single-leaved plane, the $\mu$-plane. Hence we infer (1) that the deficiency of $S$ is 0 ; (2) that $x$ is a rational function of $\mu$; for, because of the conformal relation between

* If these conditions, in a special case, should not happen to be fulfilled, $\lambda$ may be replaced through a suitable linear transformation by $\bar{\lambda}$, so that they will now be fulfilled. 
the surface $S$ and the $\mu$-plane, $x$ behaves in every region of the $\mu$-plane like a rational function; (3) that $y$ is likewise a rational function of $\mu$; for the Riemann surface that represents $x$ as a function of $y$ is conformally related to $S$ and hence to the $\mu$-plane, and $y$ behaves therefore in every region of the $\mu$-plane like a rational function; (4) that $\mu$ is a rational function of $\lambda$; this can also be inferred easily from the conformal representation, but as it follows immediately from equations (1) and (4), we will not insist on this method.

Thus all of the statements of $\$ 2$ have been proven.

$$
\text { Example. } \quad \begin{aligned}
x & =\frac{\left(\lambda^{4}+\lambda^{-4}+14\right)^{3}}{108\left(\lambda^{4}+\lambda^{-4}-2\right)^{2}}=\frac{4\left(\mu^{2}-\mu+1\right)^{3}}{27(\mu-1)^{2}} \\
y & =-\left(\frac{\lambda^{2}-1}{2 \lambda}\right)^{2}=\mu \\
\mu & =-\frac{1}{4}\left(\lambda-\lambda^{-1}\right)^{2}
\end{aligned}
$$

See Klein-Fricke, Modulfunctionen, vol. I. p. 75, for the division of the $\lambda$-plane. The $\rho=4$ regions $N, N^{\prime}, N^{\prime \prime}, N^{\prime \prime \prime}$ appear in the figure on p. 80. The notation $\lambda, \mu$ is there just the reverse of that in this paper.

HARVARD UNIVERSITY,

Cambridge, Mass., December, 1895.

\section{NOTES ON THE EXPRESSION FOR A VELOCITY- POTENTIAL IN TERMS OF FUNCTIONS OF LA- PLACE AND BESSEL.}

BY PROFESSOR JAMES MCMAHON.

1. Differential equation for $\psi$. The partial differential equation to be satisfied by a velocity-potential in an elastic fluid is, in rectangular coördinates, $*$

$$
\frac{\partial^{2} \psi}{\partial x^{2}}+\frac{\partial^{2} \psi}{\partial y^{2}}+\frac{\partial^{2} \psi}{\partial z^{2}}=\frac{1}{a^{2}} \frac{\delta^{2} \psi}{\partial t^{2}},
$$

in which $a^{2}=$ pressure/ density, and $\psi(x, y, z, t)$ is a function whose derivatives as to $x, y, z$ give the velocity-components of the fluid particle that occupies the position $(x, y, z)$ at time $t$.

2. Particular solution in polar coördinates. When (1) is transformed to polar coördinates $r, \theta, \varphi$, it can, as shown in

\footnotetext{
* RAy LeIGH, Theory of Sound, vol. II., p. 15.
} 